Surgery for

Congenital Heart

Disease

\title{
Mitral valve replacement in infants and children 5 years of age or younger: Evolution in practice and outcome over three decades with a focus on supra-annular prosthesis implantation
}

\author{
Elif Seda Selamet Tierney, MD, ${ }^{a, c}$ Frank A. Pigula, MD, ${ }^{b, d}$ Charles I. Berul, MD, ${ }^{a, c}$ James E. Lock, MD, ${ }^{a, c}$
} Pedro J. del Nido, MD, ${ }^{\text {b,d }}$ and Doff B. McElhinney, MD ${ }^{a, c}$

丹 Supplemental material is available online.
From the Departments of Cardiology and Cardiac Surgery, ${ }^{\mathrm{b}}$ Children's Hospital, and the Departments of Pediatrics ${ }^{\mathrm{c}}$ and Surgery, ${ }^{\mathrm{d}}$ Harvard Medical School, Boston, Mass.

Received for publication Oct 19, 2007; revisions received Dec 11, 2007; accepted for publication Dec 14, 2007.

Address for reprints: Doff B. McElhinney, MD, Department of Cardiology, Children's Hospital, 300 Longwood Ave, Boston, MA 02115 (E-mail: doff.mcelhinney@cardio. chboston.org)

J Thorac Cardiovasc Surg 2008;136:954-61 $0022-5223 / \$ 34.00$

Copyright $(0) 2008$ by The American Association for Thoracic Surgery

doi:10.1016/j.jtcvs.2007.12.076
Objective: Successful mitral valve replacement in young children is limited by the lack of small prosthetic valves. Supra-annular prosthesis implantation can facilitate mitral valve replacement with a larger prosthesis in children with a small annulus, but little is known about its effect on the outcomes of mitral valve replacement in young children.

Methods: One hundred eighteen children underwent mitral valve replacement at 5 years of age or younger from 1976-2006. Mitral valve replacement was supra-annular in $37(32 \%)$ patients.

Results: Survival was $74 \% \pm 4 \%$ at 1 year and $56 \% \pm 5 \%$ at 10 years but improved over time (10-year survival of $83 \% \pm 7 \%$ from 1994-2006). Factors associated with worse survival included earlier mitral valve replacement date, age less than 1 year, complete atrioventricular canal, and additional procedures at mitral valve replacement, but not supra-annular mitral valve replacement. As survival improved during our more recent experience, the risks of supra-annular mitral valve replacement became apparent; survival was worse among patients with a supra-annular prosthesis after 1991. A pacemaker was placed in $18(15 \%)$ patients within 1 month of mitral valve replacement and was less likely in patients who had undergone supra-annular mitral valve replacement. Among early survivors, freedom from redo mitral valve replacement was $72 \% \pm 5 \%$ at 5 years and $45 \% \pm 7 \%$ at 10 years. Twenty-one patients with a supra-annular prosthesis underwent redo mitral valve replacement. The second prosthesis was annular in 15 of these patients and upsized in all but 1, but 5 required pacemaker placement for heart block.

Conclusions: Supra-annular mitral valve replacement was associated with worse survival than annular mitral valve replacement in our recent experience. Patients with supra-annular mitral valve replacement were less likely to have operative complete heart block but remained at risk when the prosthesis was subsequently replaced.

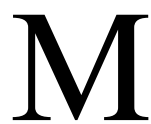

itral valve replacement (MVR) in infants and young children is an uncommon operation that historically has carried a higher mortality and worse long-term outcome than MVR in older children. ${ }^{1-13}$ One of the limiting factors in successful MVR in young children has been the lack of appropriately sized 


$$
\begin{aligned}
& \text { Abbreviations and Acronyms } \\
& \text { AV }=\text { atrioventricular } \\
& \text { LA }=\text { left atrium } \\
& \text { MR }=\text { mitral regurgitation } \\
& \text { MS }=\text { mitral stenosis } \\
& \text { MV }=\text { mitral valve } \\
& \text { MVR }=\text { mitral valve replacement } \\
& \text { PA }=\text { pulmonary artery }
\end{aligned}
$$

prosthetic valves. In certain circumstances, a mitral valve (MV) prosthesis is implanted in a supra-annular position (ie, in the left atrium [LA] above the MV annulus). Although this technique allows for a larger prosthesis to be placed, it can compromise LA volume and compliance and consequently contribute to LA hypertension in the absence of prosthetic valve obstruction. ${ }^{14}$ There are few studies with long-term follow-up after MVR in young children, and almost no published information on early or late results of supra-annular MVR. ${ }^{3,14,15}$ In particular, despite the wellcharacterized hemodynamic abnormalities that can result from supra-annular MVR, ${ }^{14}$ little is known about either early or late outcome after supra-annular MVR or about the potential for subsequent replacement of the prosthesis in an annular implant position.

\section{Materials and Methods}

This study was approved by the Children's Hospital Boston Committee on Clinical Investigation.

\section{Patients}

Patients who underwent MVR at Children's Hospital Boston between 1976-2006 were ascertained from the database of the cardiovascular program. Patients were excluded if age at MVR was greater than 5 years (60 months), if a prior MVR had been performed elsewhere, or if the MV did not support the systemic ventricle in a biventricular circulation at the time of MVR.

\section{MVR}

Indications for MVR, decisions regarding repair versus replacement, and prosthesis type varied over time according to clinical judgment and current practice and technology. Prosthesis type, size, and implant position were at the discretion of the surgeon. Anticoagulation therapy also varied over time, and was generally in keeping with prevailing practice.

\section{Data Analysis}

The primary outcome was survival over time after MVR. Secondary outcomes included early post-MVR survival (for at least 30 days and discharge from the hospital), freedom from redo MVR, freedom from placement of a permanent pacemaker, and freedom from other prosthesis-related complications. Analysis of freedom from permanent pacemaker placement only included patients who survived at least 2 weeks after MVR (ie, need for permanent pacing might have been indeterminate in patients who died within 2 weeks of
MVR) and did not have a pacemaker before MVR (ie, not at risk). Independent variables analyzed for association with outcomes included date of MVR (divided into 5-year periods, decades, and 3 periods optimized to discrimination of survival over time: 1976-1982, 1983-1993, and 1994-2006), age, weight, weight percentile for age, underlying cardiac diagnosis, indication for MVR (mitral stenosis [MS], mitral regurgitation [MR], or MS and MR), associated anomalies, prior and concurrent interventions, use of a supra-annular versus annular prosthesis, prosthesis size, and the ratio of prosthesis diameter to patient weight. Time-dependent outcomes were assessed with Kaplan-Meier analysis and multivariable Cox regression analysis. For comparison of continuous or categorical variables between groups, independent-samples $t$ tests and $\chi^{2}$ analysis were used, respectively. For comparison of preoperative and postoperative data within patients, paired $t$ test analysis was used. Multivariable analysis of discrete outcomes was performed by using multiple logistic regression. Data are presented as means \pm standard deviation or medians (range).

\section{Results}

\section{Patients}

From 1976-2006 inclusive, 118 children underwent MVR at 5 years of age or younger. The median age was 16.3 months (range, 3 days-60 months), with 50 (42\%) patients 1 year of age or younger and $84(71 \%)$ patients 2 years of age or younger. Approximately half as many patients underwent MVR between 1996 and $2006(n=25)$ as during each of the prior decades $(1976-1985, \mathrm{n}=48 ; 1986-1095, \mathrm{n}=45)$. In contrast, the number of patients 5 years of age or younger who underwent MV repair and balloon dilation of the MV increased substantially during this period (Figure 1). There

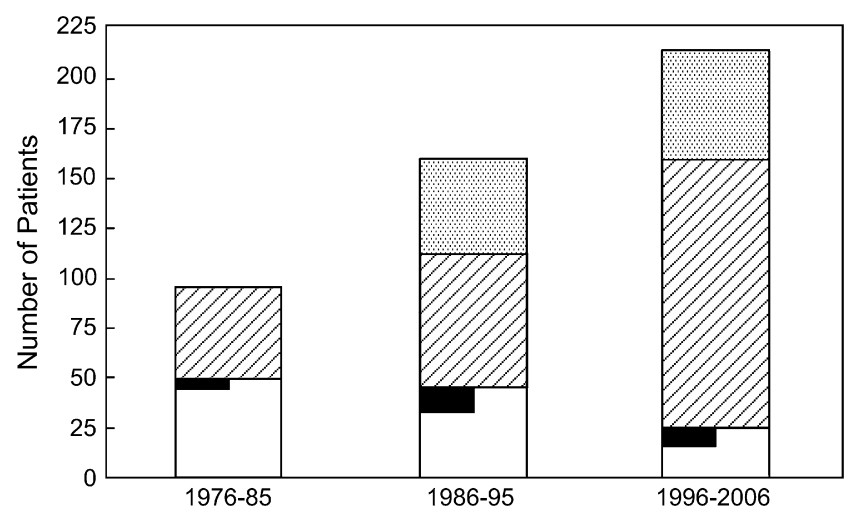

Figure 1. During the time period covered by this study, the number of mitral valve replacement (MVR) procedures (white bars) has decreased, whereas the numbers of surgical mitral valve (MV) repair (diagonally hatched bars) and balloon MV dilation (stippled bars) procedures have increased. Among patients undergoing MVR, those in whom a prior MV repair had been performed, not including initial repair of atrioventricular (AV) canal (whether complete, partial, or transitional), are shown in black. The number of patients undergoing surgical MV repair does not include patients having initial repair of AV canal. 
TABLE 1. Primary diagnosis involving the MV in 118 patients undergoing MVR at 5 years of age or younger

\begin{tabular}{lc} 
Congenital MS & $37(31 \%)$ \\
Congenital MR & $15(13 \%)$ \\
Complete or transitional AV canal & $42(36 \%)$ \\
$\quad$ With tetralogy of Fallot & $6(5 \%)$ \\
Partial AV canal (primum atrial septal defect, cleft MV) & $10(8 \%)$ \\
Isolated cleft MV & $4(3 \%)$ \\
Endocarditis & $3(3 \%)$ \\
Marfan syndrome & $2(2 \%)$ \\
Tetralogy of Fallot with associated abnormal MV & $2(2 \%)$ \\
MV prolapse with MR (chronic anemia) & $1(1 \%)$ \\
Anomalous left coronary artery from the PA & $1(1 \%)$ \\
Traumatic MR (Konno procedure) & $1(1 \%)$ \\
\hline
\end{tabular}

$M V$, Mitral valve; $M V R$, mitral valve replacement; $M S$, mitral stenosis; $M R$, mitral regurgitation; $A V$, atrioventricular; $P A$, pulmonary artery.

was no difference in age at MVR between decades. The median weight at the time of MVR was $7.5 \mathrm{~kg}$ and ranged from 3.0 to $18.0 \mathrm{~kg}$. Weight for age at the time of MVR was less than the first percentile in $65(55 \%)$ patients and less than the third percentile in $87(74 \%)$ patients.

Underlying MV-related diagnoses are summarized in Table 1. The indication for MVR was MR in $72(61 \%)$ patients, MS in $20(17 \%)$ patients, and a combination of MR and MS in 26 (22\%) patients. There was a shift in indications for MVR over time, such that patients were less likely to undergo MVR for MS and more likely to undergo MVR for mixed MR and MS during the latter part of our experience (Figure E1). This trend is probably due to an increased number of patients undergoing balloon or surgical mitral valvuloplasty for MS who developed MR, patients who had secondary MS after undergoing surgical mitral valvuloplasty for MR, or both. There was no difference in age at MVR according to hemodynamic indication. Mean pulmonary artery (PA) pressure measured at catheterization before MVR ( $\mathrm{n}=$ 80) was $46.6 \pm 19.2 \mathrm{~mm} \mathrm{Hg}$, mean LA pressure was $20.7 \pm$ $7.2 \mathrm{~mm} \mathrm{Hg}$, and indexed pulmonary vascular resistance index was $7.8 \pm 6.0 \mathrm{~mm} \mathrm{Hg} \cdot \mathrm{L}^{-1} \cdot \mathrm{min}^{-1} \cdot \mathrm{m}^{-2}$.

Cardiovascular interventions were performed before MVR in 98 (83\%) patients (Table 2). Prior interventions for MS or MR, not including initial repair of an atrioventricular (AV) canal (any type, including partial), were performed in $42(36 \%)$ patients. Prior MV procedures were performed a median of 3.0 months before MVR (range, 2 days-33 months). Among 44 patients who previously underwent repair of an AV canal, MVR was performed a median of 1.6 months (range, 1 day -42 months) after the original repair.

\section{MVR}

MV repair was attempted at the same operation as MVR in 12 $(10 \%)$ patients before the decision was made to replace the valve. Additional procedures were performed in 39 patients (Table 2).

\section{TABLE 2. Prior and simultaneous cardiovascular interventions}

\begin{tabular}{lc}
\hline Prior interventions & \\
Any intervention & $98(83 \%)$ \\
Surgical intervention & $83(70 \%)$ \\
Catheter intervention & $30(25 \%)$ \\
MV procedure & $42(36 \%)$ \\
MV surgery (not including initial AV canal repair) & $26(22 \%)$ \\
MV balloon valvuloplasty & $18(15 \%)$ \\
Supravalvar mitral ring resection & $6(5 \%)$ \\
Surgical or transcatheter intervention & $22(19 \%)$ \\
for aortic coarctation & \\
Surgical or transcatheter intervention & $12(8 \%)$ \\
$\quad$ for aortic stenosis & \\
Intervention for subaortic stenosis & $7(6 \%)$ \\
Repair of complete AV canal & $35(30 \%)$ \\
Repair of partial AV canal & $9(8 \%)$ \\
Ventricular septal defect closure & $10(8 \%)$ \\
Tetralogy of Fallot repair & $8(7 \%)$ \\
Other & $9(7 \%)$ \\
Additional interventions at the time of MVR & \\
Any simultaneous intervention & $39(33 \%)$ \\
Aortic valve repair or replacement & $8(7 \%)$ \\
Intervention for subaortic stenosis & $12(10 \%)$ \\
Repair of complete AV canal & $7(6 \%)$ \\
Ventricular septal defect closure & $8(7 \%)$ \\
Left atrial augmentation & $2(2 \%)$ \\
Tricuspid valve repair & $2(2 \%)$ \\
Other & $7(6 \%)$ \\
\hline
\end{tabular}

$M V$, Mitral valve; $A V$, atrioventricular; $M V R$, mitral valve replacement.

The types and sizes of MV prostheses are summarized in Table E1. Small-diameter dura mater valves were used in the earliest years of our experience, and the current preference is to implant low-profile St Jude HP prostheses. The prosthesis was placed in the supra-annular position in 37 $(32 \%)$ patients. By means of univariable analysis, supra-annular prosthesis placement was associated with age younger than 2 years at MVR (odds ratio [OR], 7.0; 95\% CI, 2.0-24; $P=.001)$, lower weight $(6.6 \pm 2.0$ vs $8.6 \pm 3.5 \mathrm{~kg}, P=$ $.002)$, an underlying diagnosis of congenital MS (OR, 16.8; 95\% CI, 6.4-44.1; $P<.001$ ), and MS (with or without associated MR) as an indication for MVR (OR, 10.9; 95\% CI, 4.3-27.2; $P<.001$ ). By means of multivariable logistic regression, lower weight and congenital MS were independently associated with increased likelihood of supra-annular MVR. The median prosthesis size was smaller in patients receiving a supra-annular MVR than in those in whom the prosthesis was annular (17 vs $19 \mathrm{~mm}, P=.005$ ), but there was no difference when the analysis was limited to patients younger than 2 years of age, which included all but 3 of the patients with a supra-annular implant. As depicted in Figure 2, supra-annular MVR was performed in $49 \%$ of cases between 1986-1995, which was significantly more often than in earlier or later years, when it was 


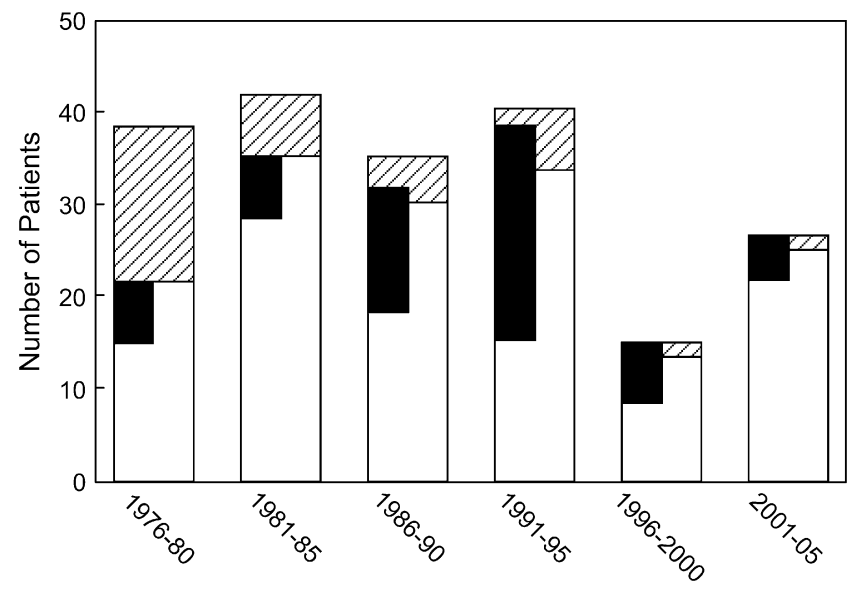

Figure 2. Bar graph depicting the number of mitral valve replacement (MVR) procedures broken down by early survivors (white bars) and early deaths (hatched bars) in 5-year blocks (the most recent block, 2001-2006, is 6 years). Patients in whom a supra-annular MVR was performed are depicted with black bars, which are accurately aligned across bars for early survivors and early deaths.

performed in $21 \%$ of cases $(\mathrm{OR}, 3.7 ; 95 \% \mathrm{CI}, 1.7-8.4$; $P=.001)$.

A number of methods of indexing prosthesis size to patient size were calculated; ultimately, the ratio of prosthesis size (diameter of the sewing ring) to patient weight was found to be most useful. The median prosthesis size/weight ratio was 2.4 (95\% CI, 1.2-5.7), with an almost linear inverse relationship between age and size/weight ratio. Among patients 1 year of age or younger at MVR, all had a prosthesis size/weight ratio of greater than 2 , and with only a few exceptions, patients older than 1 year of age had a prosthesis size/ weight ratio of less than 3 .

\section{Outcomes}

Survival. Follow-up data were available for all but 6 patients, who were referred from elsewhere and discharged from the hospital alive but had no subsequent data available. Early mortality (30 days or before hospital discharge) was $19 \%(\mathrm{n}=23)$ and decreased significantly from decade to decade (Figure 2). Factors associated with increased risk of early mortality included surgical intervention during the earliest portion of our experience (1976-1982: 13/32 [41\%] compared with 1983-1993: 8/49 [16\%] or 1994-2006: $2 / 37$ [5\%]; $P<.001$ ), age less than 1 year at MVR (OR, 2.5; 95\% CI, 1.0-6.5; $P=.045$ ), and other procedures at the time of MVR (OR, 3.4; 95\% CI, 1.3-8.8; $P=.008$ ). Among 50 patients 1 year of age or younger at MVR, all 14 early deaths occurred in patients with a prosthesis size/weight ratio greater than $3(P=.02)$. Overall, supra-annular MVR was not associated with early mortality. However, supra-annular
MVR was strongly associated with increased risk of early death during the most recent half of our experience ( $24 \%$ early mortality since 1991 compared with $5 \%$ among patients receiving an annular MVR), with 5 of 6 early deaths during this period in patients with a supra-annular implant. In contrast, none of the 14 early deaths during the first decade were in patients with a supra-annular implant.

Twenty-three patients died during follow-up: 4 after redo MVR, 2 after heart or heart-lung transplantation, 4 from arrhythmia or ventricular failure, 3 with infection, 2 with documented dysfunction or thrombosis of the MV prosthesis, 1 from right ventricular failure after surgical intervention for pulmonary valve replacement, and 7 from unknown causes. Among current survivors, 35 have been followed for 10 years or longer after MVR, 11 for 20 years or longer (6 with an initial supra-annular MVR), and 6 for 25 years or longer (4 with a supra-annular MVR).

Overall, time-related survival estimated by means of Kaplan-Meier analysis was $74 \% \pm 4 \%$ at 1 year, $62 \% \pm 5 \%$ at 5 years, $56 \% \pm 5 \%$ at 10 and 15 years, and $53 \% \pm 6 \%$ at 20 years. Survival, as determined by means of Kaplan-Meier analysis, also improved significantly over our experience, from $56 \% \pm 9 \%$ at 1 year and $46 \% \pm 9 \%$ at 5 and 10 years during the first period (1976-1982) to $92 \% \pm 5 \%$ at 1 year and $83 \% \pm 7 \%$ at 5 and 10 years during the third period (1994-2006, Figure 3). Compared with patients treated from 1976-1982, survival among patients treated between 1983-1993 was better through 5 to 7 years, but the difference was eclipsed by 10 years. There was a significant improvement in intermediate survival after 1994.

Factors associated with shorter survival over time by means of univariable and multivariable Cox regression included MVR at an earlier date (relative to 1994-2006: 1976-1982, B $=1.53, P=.01$, multivariable $P=.017$; 1983-1993, B $=1.27, P=.003$, multivariable $P=.017$ ),

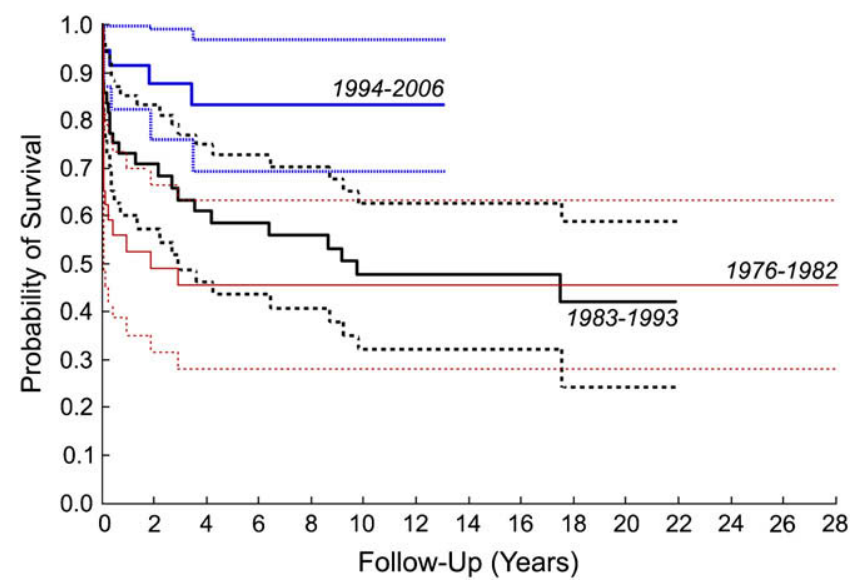

Figure 3. Kaplan-Meier survival curves for patients undergoing mitral valve repair between 1976-1982, 1983-1993, and 1994-2006. Dashed lines represent $95 \%$ confidence intervals. 
age less than 1 year at MVR $(\mathrm{B}=-0.60, P=.04$, multivariable $P=.016)$, a diagnosis of complete AV canal $(\mathrm{B}=$ $-0.78, P=.009$, multivariable $P=.001$ ), and other procedures at the time of MVR $(\mathrm{B}=-0.89, P=.001$, multivariable $P=.001)$. Notably, supra-annular MVR was not associated with time-related survival overall $(P=.53)$ but, as was the case with early mortality, was associated with worse survival over time during the most recent half of our experience. Among patients in this series undergoing MVR since 1991, 1- and 5-year survivals were $96 \%$ and $90 \%$, respectively, among those with annular MVR, and 67\% and $52 \%$, respectively, among patients with a supra-annular implant $(P=.004)$.

Survival over time was assessed for patients who survived the early post-MVR period to differentiate factors associated with perioperative and later mortality. Among early survivors, placement of a permanent pacemaker in the peri-MVR period $(\mathrm{B}=-1.4, P=.001)$, a diagnosis of complete AV canal $(\mathrm{B}=-1.01, P=.01)$, and smaller MVR prosthesis size $(\mathrm{B}=-0.23, P=.03)$ were associated with worse survival over time. By means of multivariable Cox regression, peri-MVR pacemaker placement $(P=.003)$ and smaller prosthesis size $(P=.04)$ were associated with worse survival. During the latter half of our experience, time-related survival among early survivors was worse among those with a supraannular MVR (68\% at 5 years) than those with an annular implant (94\% at 5 years, $P=.05$ ). Of 8 late deaths in patients with a supra-annular MVR, 2 occurred early after redo MVR, 2 early after heart or heart-lung transplantation, 1 from prosthesis thrombosis, 1 from ventricular failure, 1 from infection, and 1 from unknown causes. All 4 of the patients who died after reoperation had pulmonary hypertension, with a pulmonary vascular resistance index ranging from 6.4 to $19 \mathrm{~mm}$ $\mathrm{Hg} \cdot \mathrm{L}^{-1} \cdot \min ^{-1} \cdot \mathrm{m}^{-2}$ on preoperative catheterization.

Pacemaker placement. At the time of MVR, 6 patients had already had a permanent pacemaker. Another 18 patients had a pacemaker placed within 1 month of MVR for complete AV block $(n=16)$ or sinus node dysfunction $(n=2)$. An additional 10 patients had a pacemaker placed during follow-up for complete or high-grade AV block $(\mathrm{n}=8)$ or sinus node dysfunction $(n=1)$, either after redo MVR $(n=8)$ or closure of a paravalvar leak $(\mathrm{n}=1)$, and 1 for resynchronization of ventricular dysfunction unrelated to MV reintervention.

Among the 99 patients who survived at least 2 weeks after MVR and did not have a preexisting pacemaker, a diagnosis of complete AV canal (OR, 5.6; 95\% CI, 1.9-16.9; $P=.001$ ) and MVR during the first half of our experience (OR, 5.3; 95\% CI, 1.4-19.6; $P=.008$ ) were the only independent risk factors for early post-MVR pacemaker placement by means of multivariable logistic regression. By using univariable analysis, patients with a supra-annular prosthesis had a lower risk of pacemaker placement in the early postMVR period than those with an annular prosthesis (OR, $0.1 ; 95 \%$ CI, 0.01-0.73; $P=.01) ; 1$ (3\%) of 32 patients with a supra-annular prosthesis had a pacemaker placed compared with 17 (16\%) of 66 patients with an annular MVR.

Freedom from pacemaker placement among 2-week survivors was $81 \%$ at 1 month and 1 year, $78 \%$ at 5 years, and $69 \%$ at 10 years after MVR (Figure 4). By means of multivariable Cox regression, independent risk factors for shorter freedom from pacemaker placement over time were a diagnosis of complete AV canal $(\mathrm{B}=-0.85, P=.03)$ and a prosthesis/weight ratio of less than 3 at the time of $\operatorname{MVR}(\mathrm{B}=-1.2, P=.03)$.

Redo MVR and other reinterventions. Forty-four patients underwent a total of 51 redo MVR procedures during follow-up. Indications for the first redo MVR were thrombus, endocarditis, or clear prosthesis dysfunction in 9 patients; subaortic stenosis requiring surgical intervention in 4 patients ( 2 caused by prosthesis impingement); and prosthetic MS, including pannus-related obstruction, in 31 patients. Prosthesis size was increased in essentially all patients (Figure 5). As noted above, $8(18 \%)$ of these 44 patients had a permanent pacemaker placed for complete AV block early after redo MVR.

Among 95 early survivors, freedom from redo MVR was $72 \% \pm 5 \%$ at 5 years and $45 \% \pm 7 \%$ at 10 years (Figure 4 ). Factors associated with shorter freedom from redo MVR among early survivors included younger age $(\mathrm{B}=-0.37)$, smaller prosthesis size $(\mathrm{B}=-0.32, P<.001)$, and supra-annular prosthesis position $(\mathrm{B}=-0.69, P=.02)$. By means of multivariable analysis, smaller prosthesis size was the only independent predictor of shorter freedom from redo MVR. Early mortality after the first redo MVR procedure was $9 \%(4 / 44)$.

Of 37 patients who received a supra-annular prosthesis, 21 underwent redo MVR, 6 (29\%) cases of which were supraannular. In all but 1 of these patients, including all 15 who had redo MVR in the annular position, the second prosthesis was able to be upsized (Figure 5). However, among the 15

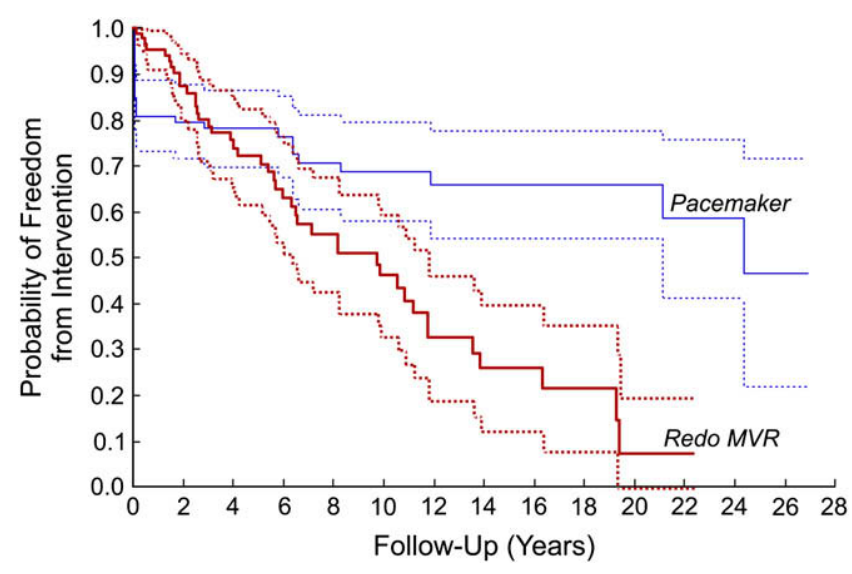

Figure 4. Kaplan-Meier curves depicting freedom from redo mitral valve replacement (MVR) and placement of a permanent pacemaker. Dashed lines represent $95 \%$ confidence intervals. 


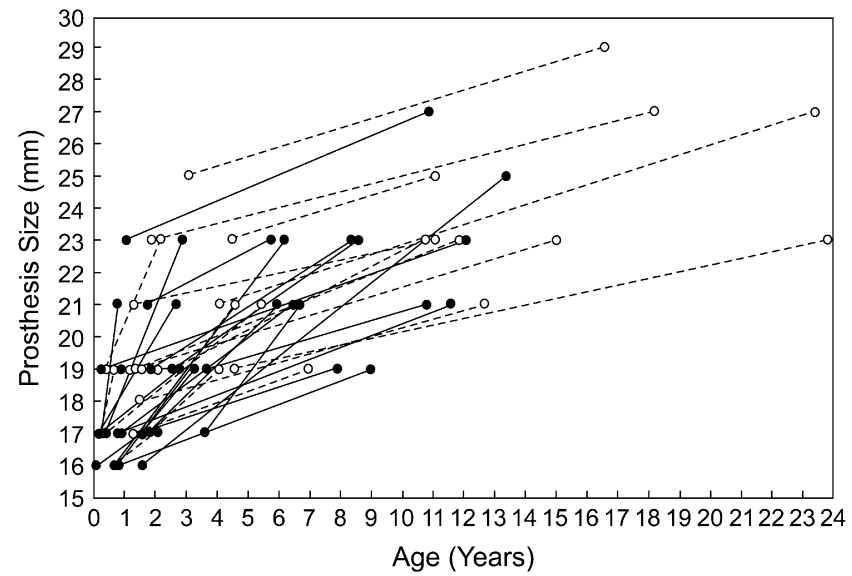

Figure 5. Line graph depicting prosthesis diameter at the time of the initial mitral valve repair (MVR) and first redo MVR in patients who initially underwent a supra-annular MVR (filled circles and lines) and those who underwent standard annular MVR (open circles and dashed lines).

patients who underwent redo MVR in the annular position after an original supra-annular MVR, 5 had complete AV block and underwent pacemaker placement after the first redo MVR. No patient who underwent an initial annular MVR had a redo prosthesis placed in the supra-annular position.

Six patients underwent 8 procedures to close paravalvar leaks, 2 in the catheterization laboratory and 6 surgically. LA augmentation was performed in 4 patients at the time of reoperation (first redo MVR in 2, second redo MVR in 1 , and subaortic stenosis resection without redo MVR in 1), including 2 who also underwent fibrous body reconstruction and MV annulus enlargement along with redo MVR and aortic valve replacement. All 4 of these patients initially had a supra-annular prosthesis.

Three patients underwent heart transplantation for ventricular failure ( 1 heart-lung transplantation), and 2 died early after transplantation. Other reinterventions during follow-up included aortic valve replacement in 5 patients, relief of subaortic stenosis in 10 patients, procedures for recurrent aortic coarctation in 3 patients, and replacement of a right ventriclePA conduit in 2 patients.

Hemodynamic status. Among patients with a supra-annular MVR initially, 21 underwent catheterization during followup, all before redo MVR or other surgical reintervention. Some of the characteristic hemodynamic findings in these patients have been reported previously. ${ }^{14} \mathrm{We}$ also found that of $11 \mathrm{pa}-$ tients with a supra-annular MVR and increased pulmonary vascular resistance index $\left(6.3-12.8 \mathrm{~mm} \mathrm{Hg} \cdot \mathrm{L}^{-1} \cdot \min ^{-1} \cdot \mathrm{m}^{-2}\right)$, all 5 who were tested with hyperoxia and inhaled nitric oxide demonstrated some degree of reactivity, with reductions in pulmonary vascular resistance index by $25 \%$ to $54 \%$, from a median of 11.5 to $7.6 \mathrm{~mm} \mathrm{Hg} \cdot \mathrm{L}^{-1} \cdot \min ^{-1} \cdot \mathrm{m}^{-2}$. Among patients who underwent redo MVR and had preoperative catheterization, pulmonary vascular resistance before reoperation was higher in those with a supra-annular prosthesis than in those without $\left(7.2 \pm 3.6 \mathrm{vs} 4.1 \pm 2.0 \mathrm{~mm} \mathrm{Hg} \cdot \mathrm{L}^{-1} \cdot \mathrm{min}^{-1}\right.$. $\left.\mathrm{m}^{-2}, P=.02\right)$.

An aneurysm of the atrialized portion of the left ventricle (between the prosthesis and native MV annulus) was observed in 2 patients (Figure E2), with no obvious consequences.

\section{Discussion}

\section{Supra-annular MVR}

Although the frequency of MVR in young patients has decreased over the past 10 to 15 years of our experience, with more aggressive approaches to MV repair for MR and MS and more frequent use of balloon mitral valvuloplasty for MS, MVR remains the only treatment option for MV dysfunction in some cases. One of the major challenges in the treatment of MV disease in infants and young children is the limitation of small prostheses for MVR. One option for implanting or maximizing the size of a mechanical MV prosthesis in small patients is to place the valve in a supra-annular position, effectively within the LA. Despite the potential benefits of a larger prosthesis in small children, supra-annular MVR has potential drawbacks, including reduction of LA volume and compliance ${ }^{14}$ and aneurysm formation in the ventricularized segment of LA between the prosthesis and annulus. ${ }^{15}$

In our experience, approximately one third of patients who underwent MVR at 5 years of age or younger received a supraannular prosthesis. Supra-annular MVR was more common in younger and smaller patients, those with congenital MS, and during the period from 1986-1995. Supra-annular MVR was associated with improved short-term survival and freedom from complete AV block compared with standard annular MVR during the early part of our experience, when survival overall was quite poor. Perhaps as a result, supra-annular MVR was used more frequently during the middle portion of our experience. As short-term survival overall improved during our more recent experience, the risks associated with supra-annular MVR became apparent because both early and intermediate survivals were worse among patients with a supra-annular prosthesis after 1991. Nevertheless, among patients who underwent successful supra-annular MVR during the earlier years of our experience, there are a number of long-term survivors who continue to do well, including 4 of the 6 patients followed for more than 25 years in this series.

Freedom from redo MVR was shorter in patients with a supra-annular prosthesis, but more than $70 \%$ of patients who underwent redo MVR after supra-annular MVR were able to have a larger prosthesis placed in the annular position. One of the most notable findings of this study was that the risk of post-MVR complete AV block requiring permanent pacemaker placement was lower in children who had a supra-annular prosthesis than in those who received an annular 
prosthesis. This finding seems reasonable, given the proximity of the MV annulus and fibrous body to the penetrating bundle. Unfortunately, also consistent with this observation, $33 \%$ of patients who underwent annular MVR after prior supra-annular MVR had AV block requiring permanent pacing after the redo MVR.

Although it is encouraging that supra-annular MVR is not associated with worse survival overall than annular MVR in infants and young children, survival trends during the most recent half of our experience better reflect the relative risk of supra-annular MVR in the current era. Whether the risks of supra-annular MVR are a function of the procedure per se or of the underlying anatomic substrate that is not amenable to annular MVR is difficult to know. However, it is likely that the effects of a supra-annular prosthesis are at least partially responsible. As reported previously, LA compliance is reduced in patients with a supra-annular MV prosthesis, which predisposes to a steep and high increase in LA pressure during ventricular systole (ie, V-wave) and consequent LA hypertension out of proportion to prosthetic MS. ${ }^{14}$ These adverse hemodynamic effects might have important clinical implications. LA and pulmonary venous hypertension might contribute to PA hypertension, which is one of the most serious potential adverse outcomes in this patient population. Generally, transprosthesis flow velocity estimated by means of Doppler echocardiographic analysis is a good indicator of LA and PA pressure. However, because the prosthetic MS gradient assessed by Doppler analysis might underrepresent the severity of LA hypertension in patients with poor LA compliance, the risk of PA hypertension might not be appreciated in these patients. Accordingly, practitioners should have a low threshold for catheterization in patients with clinical symptoms that do not appear concordant with echocardiographic findings after supra-annular MVR.

The importance of pulmonary hypertension after supraannular MVR in young children is difficult to assess from this study. However, it is noteworthy that all 4 patients with a supra-annular prosthesis who died after reoperation had increased pulmonary vascular resistance. Among the small number of patients found to have increased pulmonary vascular resistance at catheterization after supra-annular MVR, 5 were tested with inhaled nitric oxide, and all responded with a decrease (but not normalization) of the pulmonary vascular resistance index. Thus, nitric oxide is likely to be a useful adjunct in the perioperative management of children with pulmonary hypertension who undergo redo MVR.

\section{Other Findings}

Aside from the findings relating to supra-annular MVR, there are several notable results of this study. Over the past 3 decades, the approach to MV disease in infants and young children has changed substantially, with more aggressive efforts to treat MS and MR without MVR, including balloon dilation of the MV for MS and surgical MV repair for MR. ${ }^{16,17} \mathrm{MVR}$ is performed half as frequently now as it was 20 or 25 years ago, whereas the volume of surgical MV repair and balloon MV dilation procedures has grown dramatically. With these changes in practice, operative and late survivals have also continued to improve, with 1- and 10-year survivals increasing from $56 \% \pm 9 \%$ and $46 \% \pm 9 \%$ between 1976-1982 to $92 \% \pm 5 \%$ and $83 \% \pm 7 \%$ between 1994-2006. Other factors associated with worse survival, which are similar to findings of other series ${ }^{4}$ and include age less than 1 year at MVR, larger prosthesis size/patient weight ratio, and a diagnosis of complete AV canal, have not changed over this long study period. Of note, among early survivors, survival over time was worse for those who required a permanent pacemaker in the peri-MVR period.

\section{Limitations}

This study is limited by its retrospective design and inclusion of patients managed over a 30-year period. Also, it is important to acknowledge that the extent to which an MVR implant is supra-annular can vary considerably.

\section{Conclusions}

The frequency of MVR in infants and small children has decreased at our center in recent years, with a concomitant increase in the use of other surgical and interventional therapies for MV disease. Survival after MVR in children younger than 5 years of age continues to improve. In our more recent experience, supra-annular MVR is associated with worse survival over time than annular MVR. In survivors after supra-annular MVR, redo MVR can usually be performed in the annular position with appropriate prosthesis upsizing. Patients who undergo supra-annular MVR are less likely to experience operative complete AV block but remain at risk when the supra-annular prosthesis is subsequently replaced with an annular prosthesis. We believe that supra-annular MVR should be reserved for cases in which balloon or surgical valvuloplasty has failed and in which an annular implant is not feasible. In such circumstances patients typically do well in the short term but must be followed closely for occult LA and PA hypertension.

\section{References}

1. Kadoba K, Jonas RA, Mayer JE, Castaneda AR. Mitral valve replacement in the first year of life. J Thorac Cardiovasc Surg. 1990;100:762-8.

2. van Doorn C, Yates R, Tsang V, deLeval M, Elliott M. Mitral valve replacement in children: mortality, morbidity, and haemodynamic status up to medium term follow up. Heart. 2000;84:636-42.

3. Zweng TN, Bluett MK, Mosca R, Callow LB, Bove EL. Mitral valve replacement in the first 5 years of life. Ann Thorac Surg. 1989;47:720-4.

4. Caldarone CA, Raghuveer G, Hills CB, Atkins DL, Burns TL, Behrendt DM, et al. Long-term survival after mitral valve replacement in children aged $<5$ years: a multi-institutional study. Circulation. 2001;104(suppl 1):I143-7.

5. Gunther T, Mazzitelli D, Schreiber C, Wottke M, Paek SU, Meisner H, et al. Mitral-valve replacement in children under 6 years of age. Eur $J$ Cardiothorac Surg. 2000;17:426-30. 
6. Alexiou C, Galogavrou M, Chen Q, McDonald A, Salmon AP, Keeton BK, et al. Mitral valve replacement with mechanical prostheses in children: improved operative risk and survival. Eur J Cardiothorac Surg. 2001;20:105-13.

7. Erez E, Kanter KR, Isom E, Williams WH, Tam VK. Mitral valve replacement in children. J Heart Valve Dis. 2003;12:25-9.

8. Eble BK, Fiser WP, Simpson P, Dugan J, Drummond-Webb JJ, Yetman AT. Mitral valve replacement in children: predictors of longterm outcome. Ann Thorac Surg. 2003;76:853-9.

9. Raghuveer G, Caldarone CA, Hills CB, Atkins DL, Belmont JM, Moller JH. Predictors of prosthesis survival, growth, and functional status following mechanical mitral valve replacement in children aged $<5$ years, a multi-institutional study. Circulation. 2003;108(suppl II):II174-9.

10. Kojori F, Chen R, Caldarone CA, Merklinger SL, Azakie A, Williams WG, et al. Outcomes of mitral valve replacement in children: a competing-risks analysis. J Thorac Cardiovasc Surg. 2004; 128:703-9.

11. Masuda M, Kado H, Tatewaki H, Shiokawa Y, Yasui H. Late results after mitral valve replacement with bileaflet mechanical prosthesis in children: evaluation of prosthesis-patient mismatch. Ann Thorac Surg. 2004;77:913-7.
12. Vohra HA, Laker S, Stumper O, De Giovanni JV, Wright JG, Barron DJ, et al. Predicting the performance of mitral prostheses implanted in children under 5 years of age. Eur J Cardiothorac Surg. 2006;29:688-92.

13. Beierlein W, Becker V, Yates R, Tsang V, Elliott M, de Leval M, et al. Long-term follow-up after mitral valve replacement in childhood: poor event-free survival in the young child. Eur J Cardiothorac Surg. 2007;31:861-6.

14. Adatia I, Moore PM, Jonas RA, Colan SD, Lock JE, Keane JF. Clinical course and hemodynamic observations after supraannular mitral valve replacement in infants and children. J Am Coll Cardiol. 1997;29: 1089-94.

15. Barker CL, Daubeney PE, Shinebourne EA. Complications of supra-annular mitral valve placement in infants. Heart. 2005;9:e48.

16. McElhinney DB, Sherwood MC, Keane JF, del Nido PJ, Almond CS, Lock JE. Current management of severe congenital mitral stenosis: outcomes of transcatheter and surgical therapy in 108 infants and children. Circulation. 2005;112:707-14.

17. Moran AM, Daebritz S, Keane JF, Mayer JE. Surgical management of mitral regurgitation after repair of endocardial cushion defects: early and midterm results. Circulation. 2000;102(suppl III):III160-5. 


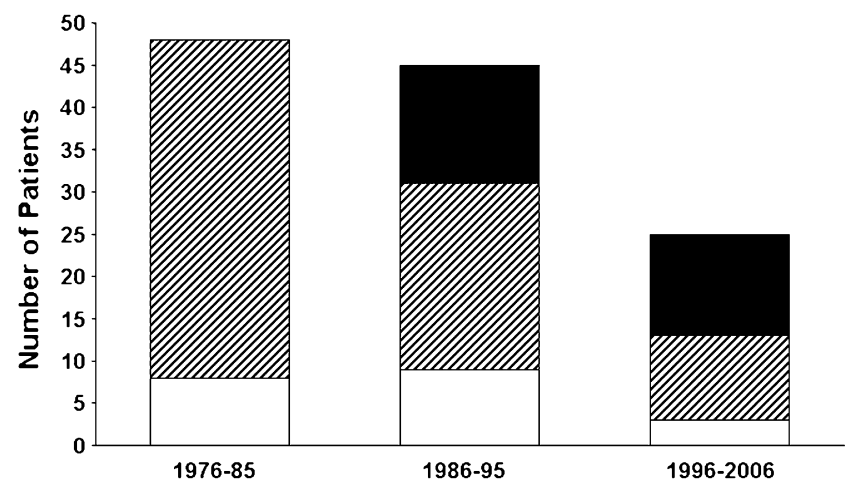

Figure E1. Mitral valve repair procedures during each decade broken down by primary indication: mitral stenosis (white bars), mitral regurgitation (hatched bars), or mixed mitral stenosis and mitral regurgitation (black bars). 

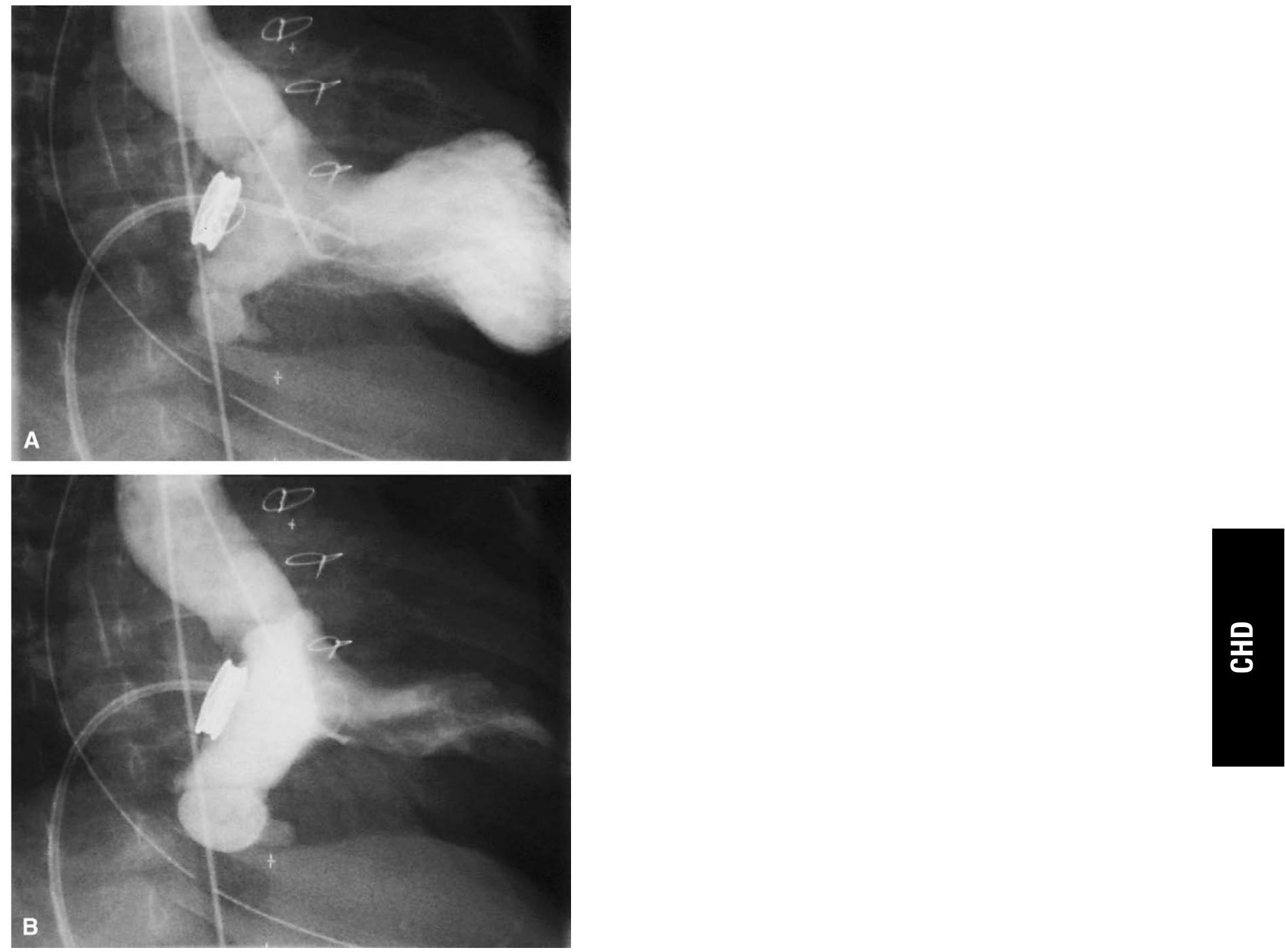

Figure E2. Images from a retrograde left ventricular angiogram during diastole $(A)$ and systole $(B)$ in a patient with an aneurysm of the ventricularized portion of the left atrium. The ventricularized portion of the left atrium between the prosthesis and the native mitral valve annulus is the densely opacified area in the systolic image. The pigtail catheter was introduced into the left ventricle retrograde, through the aortic valve. The other catheter, a balloontipped end-hole catheter, appears to be across the mitral valve prosthesis in these images but is actually in the right ventricle. 
TABLE E1. MVR prosthesis types and sizes

\begin{tabular}{lc}
\hline Prosthesis type & \\
St Jude & $49(42 \%)$ \\
Bjork-Shiley & $36(30 \%)$ \\
Carbomedics & $15(13 \%)$ \\
Porcine & $6(5 \%)$ \\
Dura mater & $6(5 \%)$ \\
St Jude-HP & $5(4 \%)$ \\
Allograft & $1(1 \%)$ \\
Prosthesis size & \\
$\leq 17 \mathrm{~mm}$ & $48(41 \%)$ \\
$18-19 \mathrm{~mm}$ & $38(32 \%)$ \\
$\geq 21 \mathrm{~mm}$ & $32(27 \%)$ \\
\hline
\end{tabular}

MVR, Mitral valve replacement. 